\title{
Perspective for Part I
}

\author{
Hiroyuki Yamagishi
}

The lungs are the primary organs of the respiratory system in vertebrates, other than fish, that function as an efficient gas exchanger. This respiratory system is physically and physiologically connected to the cardiovascular system to extract oxygen from the atmosphere and transfer it into the bloodstream and to release carbon dioxide from the bloodstream into the atmosphere. The normal pulmonary circulation, in contrast to the systemic circulation, has low pressure because of a low-resistant vascular bed that enables highly efficient gas exchange between the millions of alveoli and capillaries. Pulmonary hypertension $(\mathrm{PH})$ is a rare pulmonary arterial disease with a high mortality and morbidity although treatment options have improved in the last decades. PH is defined by a mean pulmonary artery pressure (mPAP) greater than or equal to $20 \mathrm{mmHg}$ at rest and is classified into five main subgroups based on the etiology and hemodynamic criteria. Because PH is heterogeneous at the genetic and molecular levels, a deeper understanding is required for the development of more effective therapies.

In Part I, the basic science for the development of the lungs and the pathogenesis of PH is discussed towards a better understanding of the etiology and pathophysiology of PH. Drs. Hogan and Morimoto overview the morphogenesis and epithelial development of the lung. Each alveolus in lungs is composed of epithelial and mesenchymal populations. Dr. Hogan (Chap. 2) focuses on the interesting function of Type 2 epithelial cells, as epithelial stem cells for the alveoli, capable of long-term self-renewal and differentiation into Type 1 epithelial cells that are extremely large and thin to be specialized for gas exchange. The relative contribution of the WNT, FGF, EGF, and BMP signaling pathways as well as cytokines produced by immune

\footnotetext{
H. Yamagishi $(\square)$

Division of Pediatric Cardiology, Department of Pediatrics,

Keio University School of Medicine, Tokyo, Japan

e-mail: hyamag@keio.jp

(C) The Editor(s) (if applicable) and The Author(s) 2020

T. Nakanishi et al. (eds.), Molecular Mechanism of Congenital Heart Disease

and Pulmonary Hypertension, https://doi.org/10.1007/978-981-15-1185-1_1
} 
cells to alveolar homeostasis, repair and regrowth is discussed. Dr. Morimoto (Chap. 3) focuses on Notch signaling as a reciprocal mesenchymal-epithelial interaction that controls the development of the airway branching structure. Notchmediated cell fate selection is utilized to organize three epithelial cell types during the bronchial tree is extending. As for the mesenchymal population that gives rise to endothelial cells, pericytes, several different fibroblast subpopulations, and immune cells, Dr. Uchida et al. (Chap. 8) suggest that the expression of a transcription factor Tbx4 in the lung mesenchyme may be involved in the formation of both airways and vessels in the lungs using animal experiments. It is also known that mutations of the TBX4 gene lead to PH in human. Dr. Ishizaki et al. (Chap. 9) demonstrate that pulmonary arterial smooth muscle layers, probably derived from mesenchyme of the mesodermal origin, may elongate gradually from the proximal pulmonary arterial trunk to bilateral peripheral pulmonary arteries by using a transgenic mouse line harboring the LacZ reporter gene in the specific locus. This observation supports the "distal angiogenesis" model for pulmonary arterial development.

$\mathrm{PH}$ is also known as a progressive pulmonary vascular remodeling disease. Histopathological changes in PH include an increase in smooth muscle cells in the pulmonary vasculature with muscularization of normally non-muscularized distal pulmonary arterioles. Dr. Greif's team (Chap. 4) identified novel smooth muscle cell progenitors located at the muscular-unmuscular arteriole border that have a unique molecular signature and are the source of the vast majority of pathological distal arteriole smooth muscle cells in PH. Molecular mechanisms underlying induction, proliferation, migration, and differentiation of these specialized progenitor cells are discussed. In addition to the above-described fashions to increase smooth muscle cells in the pulmonary vasculature of $\mathrm{PH}$, recent studies have demonstrated that a part of smooth muscle cells from intimal and plexiform lesions has an endothelial origin through the process of endothelial-mesenchymal transition (EMT). Dr. Ranchoux et al. (Chap. 6) describe how EMT appears to play a crucial role in $\mathrm{PH}$ progression including the underlying molecular mechanisms. As another advanced understanding for pathogenesis of PH, Drs. Chen and Raj (Chap. 7) focus on extracellular vesicles. Subsets of extracellular vesicles are microvesicles, exosomes, and apoptotic bodies. They are released from a variety of cell types and carry cargo such as proteins and microRNAs that may play an important role in the pathogenesis of $\mathrm{PH}$.

Utilizing animal models, three important findings about the pathogenesis of $\mathrm{PH}$ are also discussed in Part I. Dr. Nakanishi et al. (Chap. 11) show morphological changes of pulmonary microvasculature in the mouse model of bronchopulmonary dysplasia (BPD). As BPD is often associated with $\mathrm{PH}$, their study may provide insights into secondary PH due to pulmonary diseases. Dr. Zhang et al. (Chap. 12) identify significant increased expression of chemokine receptor type 4 (CXCR4) and some stem cell markers in PH model rats as putative mediators of pulmonary vascular remodeling. Dr. Shibata et al. (Chap. 13) demonstrate that $\mathrm{Ca}^{2+}$ signal through the inositol trisphosphate receptor $\left(\mathrm{IP}_{3} \mathrm{R}\right)$ may be involved in the pathophysiology of $\mathrm{PH}$ using $\mathrm{IP}_{3} \mathrm{R}$ knockout mice. $\mathrm{CXCR} 4$ or $\mathrm{IP}_{3} \mathrm{R}$ might be a potential candidate for new therapeutic targets of $\mathrm{PH}$. 
Finally, Dr. Clapp's group (Chap. 5) discuss about basic science for the treatment of $\mathrm{PH}$. Although there were no treatments available for $\mathrm{PH} 20$ years ago, current therapies come from four drug classes, namely, prostanoid analogs, endothelin receptor antagonists, phosphodiesterase type 5 inhibitors, and soluble guanylate cyclase stimulators, and through focused research, there are more in development. Intravenous prostacyclin, as a prostanoid analog, remains the most efficacious treatment for $\mathrm{PH}$, and several prostacyclin analogs are approved for use via different administration routes. They act as vasodilators but potently inhibit platelet aggregation, cell proliferation, and inflammation. Dr. Clapp et al. discuss how prostacyclins might rescue BMPR2 and TASK-1 dysfunction and the importance of prostanoid EP2 receptors as negative modulators of vascular tone, proliferation, and fibrosis. BMPR2 is known as the most important genetic cause of PH, and TASK-1 is a TWIK-related acid-sensitive potassium channel that, they believe, is likely to be a key target for prostacyclins. As basic research for future stem cell therapy of PH, Dr. Maeda et al. (Chap. 10) suggest that stem cell antigen-1 in the pulmonary endothelium may potentially make progenitor cell populations stay resident in adult murine lungs.

The field of pulmonary vascular biology continues to capitalize on advances in basic science. Part I includes a number of exciting advances in basic science and how these advances are impacting the patient with PH. This knowledge should be required for physicians and scientists to be translated into future clinical practice.

Open Access This chapter is licensed under the terms of the Creative Commons Attribution 4.0 International License (http://creativecommons.org/licenses/by/4.0/), which permits use, sharing, adaptation, distribution and reproduction in any medium or format, as long as you give appropriate credit to the original author(s) and the source, provide a link to the Creative Commons license and indicate if changes were made.

The images or other third party material in this chapter are included in the chapter's Creative Commons license, unless indicated otherwise in a credit line to the material. If material is not included in the chapter's Creative Commons license and your intended use is not permitted by statutory regulation or exceeds the permitted use, you will need to obtain permission directly from the copyright holder.

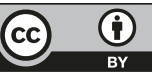

